ARTICLE

\title{
Implementation of Transient Neutron Transport Solver in ASTERIA-FBR
}

\author{
Toshihisa YAMAMOTO ${ }^{1, *}$, Hiroshi ENDO $^{1}$ \\ Tsugio YOKOYAMA ${ }^{2}$ and Masatoshi KAWASHIMA ${ }^{2}$
}

\footnotetext{
${ }^{1}$ Japan Nuclear Energy Safety Organization, TOKYU REIT Toranomon Bldg., 3-17-1 Toranomon, Minato-ku, Tokyo, 105-0001, Japan

${ }^{2}$ Toshiba Nuclear Engineering Services Corporation, 8 Shinsugita, Isogo-ku, Yokohama, Kanagawa, 235-8523, Japan
}

\begin{abstract}
Numerical methods were newly developed to perform transient calculation based on the $\alpha$-mode with a multigroup Monte Carlo code GMVP. Two methods to search $\alpha$ were investigated; one is a Newton-based-method with a use of adjoint solution, and the other is a quasi-Newton-method, which does not require the adjoint solution. The feasibility of the latter method was verified through numerical tests. The whole structure of the neutronic calculation module is shown together with some experience concerning with tally setting.
\end{abstract}

KEYWORDS: neutron transport solver, ASTERIA-FBR, transient calculation, $\alpha$-mode, $k$-mode, multigroup Monte Carlo code GMVP, Newton-based-method, adjoint solution, quasi-Newton-method, Doppler effect

\section{Introduction}

In JNES, the ASTERIA-FBR code ${ }^{1)}$ is developed as the principal tool to investigate the time dependent neutronics behaviors during core disruptive accidents of liquid metal cooled fast breeder reactors (LMFBRs). The precedent codes developed for the same purpose, such as SIMMER-III code, ${ }^{2-3)}$ have been based on deterministic methods, especially the discrete ordinate method. ${ }^{4)}$ The scheme worked fine in two-dimensions, although several drawbacks of the method have been pointed out; deterioration of solutions by the ray effect ${ }^{5)}$ with a low-order angular quadrature, and by the fixed up procedures ${ }^{6)}$ after negative flux appeared during a calculation. The possibility of producing unjustified results from SIMMER-III when the fixup is significant is reported in Ref. 7). Moreover, the extension to 3-dimensions will be indispensable in the near future and then it will be a tough challenge to ensure stable and accurate solutions using any kind of deterministic methods. These arguments hold true for SIMMER-IV code, ${ }^{8)}$ which is the direct extension of SIMMER-III to a 3-dimensional version. Considering all, the Monte Carlo method was selected as the scheme. It is worth noting that the extension of the present method to the continuous-energy Monte Carlo method, which can exactly treat the self-shielding effect of heavy nuclides, is extremely easy except the computation time issue.

In the solver, time dependence of the flux is represented by a multiplication of an amplitude function and a shape function using the quasi-static approximation ${ }^{9)}$ as is done in the precedent codes. The GMVP code, ${ }^{10)}$ which was developed by JAEA, is used for evaluation of the reactivity and shape function at each time step. The code, however, does not have the function of solving the ' $\alpha$-mode' problem ${ }^{11)}$ which is necessary to treat the approximation, but of solving

*Corresponding author, E-mail:yamamoto-toshihisa@jnes.go.jp

(C) 2011 Atomic Energy Society of Japan, All Rights Reserved. the ' $k$-mode' problem ${ }^{11)}$ and an external source problem. In order to avoid a laborious effort to modify the code itself, a new simple method is proposed to treat the $\alpha$-mode problem using only obtainable results from the present code. In the paper, the detailed procedure of the method is explained and its applicability to the relevant problems is discussed.

\section{Numerical Methods}

\section{The $\alpha$-Mode Calculation}

Cullen et al. made an extensive study on dynamic criticality including numerical experiments with Monte Carlo method. ${ }^{11)}$ In the paper, he concluded that 1) dynamic and alpha static ( $\alpha$-mode) criticality calculations are identical, and 2) The $k$ static ( $k$-mode) method is generally only accurate for systems that are close to critical.

The dynamic criticality calculation solves the following equation

$$
\begin{aligned}
& v^{-1} \partial \psi(\Omega, E) / \partial t+\Omega \bullet \vec{\nabla} \psi(\Omega, E)+\Sigma_{t}(E) \psi(\Omega, E) \\
& =\chi(E) \int_{E^{\prime}} v \Sigma_{f}\left(E^{\prime}\right) \psi\left(\Omega^{\prime}, E^{\prime}\right) d \Omega^{\prime} d E^{\prime} \\
& +\int_{E^{\prime}} \Sigma_{s}\left(\Omega^{\prime}, E^{\prime} \rightarrow \Omega, E\right) \psi\left(\Omega^{\prime}, E^{\prime}\right) d \Omega^{\prime} d E^{\prime}+S(\Omega, E),
\end{aligned}
$$

where

$$
\begin{array}{ll}
\psi(\Omega, E): & \text { angular flux, } \\
S(\Omega, E): & \text { external source, } \\
\chi(E): & \text { fission spectrum, } \\
\Sigma_{t}(E): & \text { total cross section, } \\
v \Sigma_{f}(E): & \text { production cross section, } \\
\Sigma_{s}\left(\Omega^{\prime}, E^{\prime} \rightarrow \Omega, E\right): & \text { scattering cross section, } \\
v: & \text { neutron speed. }
\end{array}
$$

The $k$-mode equation is obtained by setting $\partial \psi / \partial t=0$ and $S(\Omega, E)=0$, and introducing a new parameter $k$ such as 


$$
\begin{aligned}
& \Omega \bullet \vec{\nabla} \psi(\Omega, E)+\Sigma_{t}(E) \psi(\Omega, E)=k^{-1} \chi(E) \int_{E^{\prime}} v \Sigma_{f}\left(E^{\prime}\right) \psi\left(\Omega^{\prime}, E^{\prime}\right) d \Omega^{\prime} d E^{\prime} \\
& +\int_{E^{\prime}} \Sigma_{s}\left(\Omega^{\prime}, E^{\prime} \rightarrow \Omega, E\right) \psi\left(\Omega^{\prime}, E^{\prime}\right) d \Omega^{\prime} d E^{\prime} .
\end{aligned}
$$

As is mentioned in Ref. 11), there is no simple relationship between the $k$-mode equation and the non-critical problem that we are interested in.

In contrast, the $\alpha$-mode equation is obtained by introducing such a parameter $\alpha$ that the relation $\partial \psi / \partial t=\alpha \psi$ holds. The equation has two different forms according to the state of criticality:

$$
\begin{aligned}
& \Omega \bullet \vec{\nabla} \psi(\Omega, E)+\left[\Sigma_{t}(E)+\alpha / v\right] \psi(\Omega, E) \\
& =\chi(E) \int_{E^{\prime}} v \Sigma_{f}\left(E^{\prime}\right) \psi\left(\Omega^{\prime}, E^{\prime}\right) d \Omega^{\prime} d E^{\prime} \\
& +\int_{E^{\prime}} \Sigma_{s}\left(\Omega^{\prime}, E^{\prime} \rightarrow \Omega, E\right) \psi\left(\Omega^{\prime}, E^{\prime}\right) d \Omega^{\prime} d E^{\prime}+S(\Omega, E),
\end{aligned}
$$

for super-critical state $(\alpha>0)$, and

$$
\begin{aligned}
& \Omega \bullet \vec{\nabla} \psi(\Omega, E)+\Sigma_{t}(E) \psi(\Omega, E) \\
& =\chi(E) \int_{E^{\prime}} v \Sigma_{f}\left(E^{\prime}\right) \psi\left(\Omega^{\prime}, E^{\prime}\right) d \Omega^{\prime} d E^{\prime} \\
& +\int_{E^{\prime}} \Sigma_{s}\left(\Omega^{\prime}, E^{\prime} \rightarrow \Omega, E\right) \psi\left(\Omega^{\prime}, E^{\prime}\right) d \Omega^{\prime} d E^{\prime}+S(\Omega, E)-\alpha / v \bullet \psi(\Omega, E),
\end{aligned}
$$

for sub-critical state $(\alpha<0)$.

Eqs. (3a) and (3b) can be regarded as modified external source calculations with a pseudo absorption or an additional source $\pm \alpha / \nu \bullet \psi(\Omega, E)$.

Note also that for critical state $(\alpha=0)$, they are identical to the $k$-mode equation Eq. (2) having $k=1$.

As the state of criticality is indispensible in the $\alpha$-mode calculation, we assume the $k$-mode calculation is completed and available as initial guess for the final solution of Eq. (3a) or (3b).

In super-critical state, for example, we solve the following equation instead of Eq. (3a) in order that neutron balance be maintained with an arbitrary value of $\alpha$

$$
\begin{aligned}
& \Omega \bullet \vec{\nabla} \psi(\Omega, E)+\left[\Sigma_{t}(E)+\alpha / v\right] \psi(\Omega, E)=\chi(E) \lambda \int_{E^{\prime}} v \Sigma_{f}\left(E^{\prime}\right) \psi\left(\Omega^{\prime}, E^{\prime}\right) d \Omega^{\prime} d E^{\prime} \\
& +\int_{E^{\prime}} \Sigma_{s}\left(\Omega^{\prime}, E^{\prime} \rightarrow \Omega, E\right) \psi\left(\Omega^{\prime}, E^{\prime}\right) d \Omega^{\prime} d E^{\prime},
\end{aligned}
$$

where the source term can be neglected.

Our interest now is the strategy to find an $\alpha$ that makes the $\lambda$ as close to unity as possible making use of the $k$-mode solution.

\section{Derivation of Newton-Method-Based Formula}

The $k$-mode equation can be rewritten in more simplified form with kernel matrices $A$ and $F$ as

$$
A \phi=\lambda F \phi,
$$

where

$$
\begin{aligned}
& \phi=\phi(E)=\int \psi(\Omega, E) d \Omega, \\
& A=L+\Sigma_{t}(E)-\int_{E^{\prime}} \Sigma_{s}\left(E^{\prime} \rightarrow E\right) d E^{\prime}, \\
& L \equiv \int \Omega \bullet \vec{\nabla} \psi(\Omega, E) d \Omega / \int \psi(\Omega, E) d \Omega,
\end{aligned}
$$

$$
\begin{aligned}
& F=\chi(E) \int_{E^{\prime}} v \Sigma_{f}\left(E^{\prime}\right) d E^{\prime} \\
& \lambda=k^{-1} .
\end{aligned}
$$

If $\lambda$ and $\phi$ are expanded as power series of $\alpha$ :

$$
\begin{aligned}
& \lambda=\lambda_{(0)}+\alpha \lambda_{(1)}+\alpha^{2} \lambda_{(2)}+\ldots=\sum_{n=0}^{\infty} \alpha^{n} \lambda_{(n)}, \\
& \phi=\phi_{(0)}+\alpha \phi_{(1)}+\alpha^{2} \phi_{(2)}+\ldots=\sum_{n=0}^{\infty} \alpha^{n} \phi_{(n)},
\end{aligned}
$$

then Eq. (1) gives rise to the infinite system of equations

$$
\begin{aligned}
& A \phi_{(0)}=\lambda_{(0)} F \phi_{(0)}, \\
& A \phi_{(1)}=\lambda_{(0)} F \phi_{(1)}+\left[\lambda_{(1)} F-\operatorname{diag}\left\{v^{-1}\right\}\right] \phi_{(0)}, \\
& A \phi_{(2)}=\lambda_{(0)} F \phi_{(2)}+\lambda_{(2)} F \phi_{(0)}+\left[\lambda_{(1)} F-\operatorname{diag}\left\{v^{-1}\right\}\right] \phi_{(1)}, \\
& A \phi_{(n)}=\sum_{j \neq n-1}^{n} \lambda_{(n-j)} F \phi_{(j)}+\left[\lambda_{(1)} F-\operatorname{diag}\left\{v^{-1}\right\}\right] \phi_{(n-1)}
\end{aligned}
$$

where $\operatorname{diag}\{\bullet\}$ denotes diagonal matrix.

Equations (7)-(9) have the form of the Fredholm's integral equation of the second kind. We should note that the variational approach using Eqs. (5) is not practical. It involves direct solution of Eqs. (7)-(9) of which convergence of the Neumann expansion is extremely slow because the norm $\left\|F A^{-1}\right\|$ is close to unity in most cases.

Instead, we use the adjoint approach. Let us write the adjoint equation of Eq. (6) as

$$
A^{+} \phi^{+}=\lambda_{(0)} F^{+} \phi^{+} .
$$

For any vector $\mathrm{x}$, the following relation holds

$$
\left\langle\phi^{+},\left(A-\lambda_{(0)} F\right) x\right\rangle=\left\langle\left(A^{+}-\lambda_{(0)} F^{+}\right) \phi^{+}, x\right\rangle=\langle 0, x\rangle=0 .
$$

where $\langle\bullet\rangle$ denotes integration over space, energy.

Multiplying $\phi^{+}$from left and integrating Eq. (6), we obtain

$$
\lambda_{(0)}=\left\langle\phi^{+}, A \phi_{(0)}\right\rangle /\left\langle\phi^{+}, F \phi_{(0)}\right\rangle,
$$

which is the definition of $k$-mode eigenvalue. The same procedure to Eq.(7) gives rise to

$$
\left\langle\phi^{+}, A \phi_{(1)}\right\rangle=\left\langle\phi^{+}, \lambda_{(0)} F \phi_{(1)}\right\rangle+\left\langle\phi^{+},\left[\lambda_{(1)} F-\operatorname{diag}\left\{v^{-1}\right\}\right] \phi_{(0)}\right\rangle
$$

Then, the first order perturbation on eigenvalue is obtained as

$$
\lambda_{(1)}=\left\langle\phi^{+}, \operatorname{diag}\left\{v^{-1}\right\} \phi_{(0)}\right\rangle /\left\langle\phi^{+}, F \phi_{(0)}\right\rangle .
$$

The same procedure to Eq. (8) gives rise to

$$
\begin{aligned}
& \left\langle\phi^{+}, A \phi_{(2)}\right\rangle=\left\langle\phi^{+}, \lambda_{(0)} F \phi_{(2)}\right\rangle+\left\langle\phi^{+}, \lambda_{(2)} F \phi_{(0)}\right\rangle \\
& +\left\langle\phi^{+},\left[\lambda_{(1)} F-\operatorname{diag}\left\{v^{-1}\right\}\right] \phi_{(1)}\right\rangle
\end{aligned}
$$

Then, the second order perturbation is obtained.

$$
\lambda_{(2)}=\left\lfloor\left\langle\phi^{+}, \operatorname{diag}\left\{v^{-1}\right\} \phi_{(1)}\right\rangle-\lambda_{(1)}\left\langle\phi^{+}, F \phi_{(1)}\right\rangle\right] /\left\langle\phi^{+}, F \phi_{(0)}\right\rangle
$$




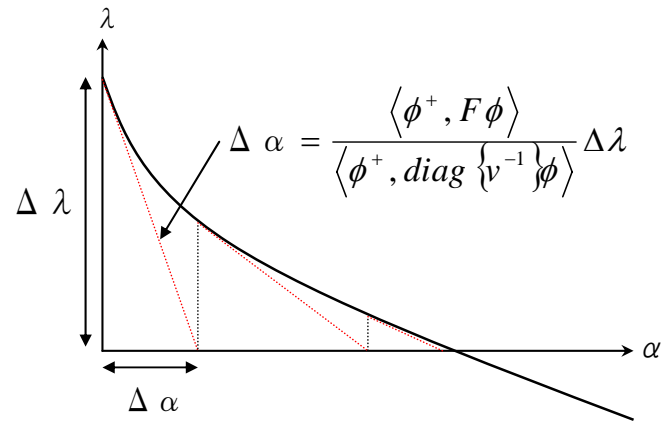

Fig. 1 The Newton-method-based procedure

As $\lambda_{(1)}$ corresponds to the gradient of the tangential line on the $\alpha-\lambda$ curve, guess of $\alpha$ that gives $\lambda \rightarrow 1$ is obtained using the Newton method (Fig. 1). The procedure is described as follows

$$
\begin{aligned}
& \alpha_{n}=\alpha_{n-1}+\Delta \alpha_{n}, \\
& \Delta \alpha_{n}=\left\langle\phi_{n}^{+}, F \phi_{n}\right\rangle /\left\langle\phi_{n}^{+}, \operatorname{diag}\left\{v^{-1}\right\} \phi_{n}\right\rangle \Delta \lambda_{n}, \\
& \Delta \lambda_{0}=\lambda_{(0)}-1 .
\end{aligned}
$$

\section{Improved Quasi-Newton Method}

The Newton-method-based procedure requires renewed adjoint flux at each trial. In order to save calculation time, an alternative "adjoint-free" method is introduced. If the $\lambda-\alpha$ curve is amply smooth, the gradient of a particular point on the curve is well approximated by the gradient of the two adjacent points located at the same distance, but in opposite direction, from the point. This assumption gives rise to the following "adjoint-free” procedure

$$
\begin{aligned}
& \left.\frac{\partial \lambda}{\partial \alpha}\right|_{\alpha_{3}} \approx \frac{\lambda\left(\alpha_{2}\right)-\lambda\left(\alpha_{1}\right)}{\alpha_{2}-\alpha_{1}}, \quad \alpha_{3}=\frac{\alpha_{1}+\alpha_{2}}{2}, \\
& \alpha_{n}=\alpha_{n-1}+\Delta \alpha_{n}, \\
& \Delta \alpha_{n}=\left(\alpha_{n}-\alpha_{n-1}\right) /\left(\lambda_{n}-\lambda_{n-1}\right) \times \Delta \lambda_{n}, \\
& \Delta \lambda_{0}=\lambda_{(0)}-1 .
\end{aligned}
$$

Hereafter, we call this the Quasi-Newton-method-based procedure (Fig. 2).

Equation (16c) has a drawback that both the denominator and numerator approach to zero for a large $n$. Thus an additional care must be taken to avoid the numerical instability, such as cutoff of the procedure when $n$ reaches to a maximum criterion.

\section{Sample Calculations}

\section{(1) Toy Problems}

In order to compare the performance of the above two methods, the following analytical functions were selected to represent the $\alpha-\lambda$ curves:

$$
\lambda-1=f_{n}(\alpha)=(1-\alpha)^{n+1}-3^{-n},
$$

where $n=1, . ., 4$.

Equation (17) is a series of concave curves whose common solution is $\alpha=2 / 3$, and the change in its gradient is

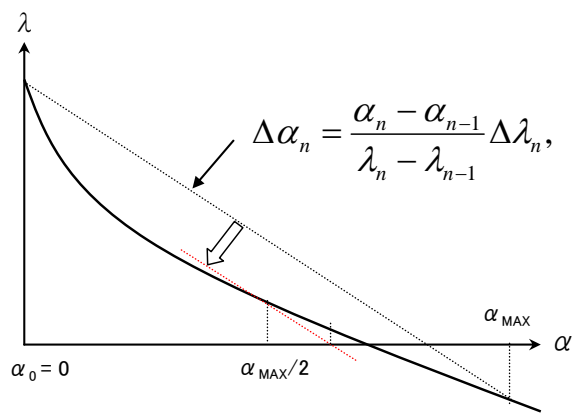

Fig. 2 The Quasi-Newton-method-based procedure

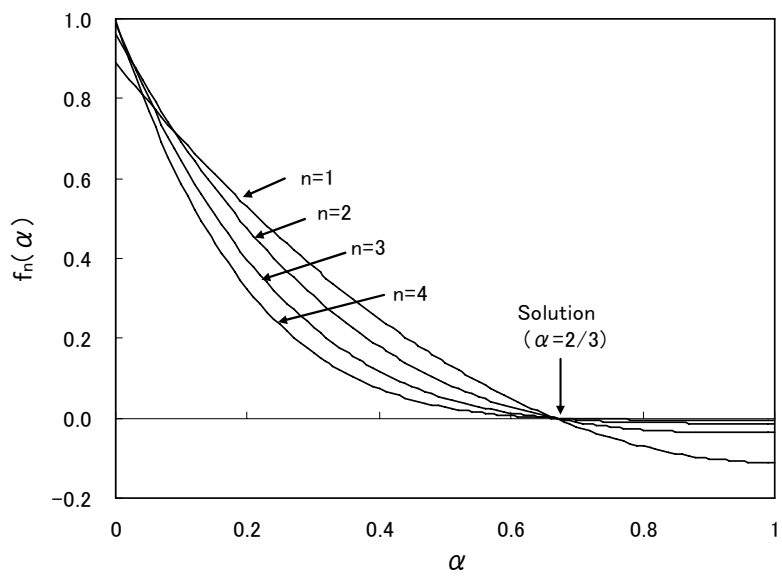

Fig. 3 Analytical functions used in the toy problems

steeper as $n$ becomes larger (Fig. 3).

Figure 4 shows the comparison of convergence performance of the two methods. As the Newton method requires the adjoint calculation at each trial, the required computation time was assumed as double as the quasi-Newton method. The Newton method reveals quick convergence as the trial proceeds, which is known as "super-linear convergence". In contrast, the convergence of the quasi-Newton method is almost linear. The Newton method overwhelms the quasi-Newton method when the $\alpha-\lambda$ curves is nearly linear (Fig. 4(a)), while the latter may be more suitable when it is not the case(Fig. 4(b)). It may worth noting that the required precision of the solution is limited to few digits in actual Monte Carlo calculations due to statistical errors, as will be seen later. Taking all into consideration, we concluded that the quasi-Newton method is superior.

(2) Monte Carlo Problem

The quasi-Newton method was applied to a small test problem which models a prototype fast reactor. A 70-group cross section set was used in the calculation to compare the difference in neutron spectrum between the two modes.

The $k$-mode eigenvalue was 1.0635 and the final $\alpha$ was $2.29 \times 10^{5}$ starting iterations from the initial value $\alpha=0$.

Figure 5 shows the convergence performance of the $\alpha$ parameter. A smooth convergence was observed in the first four iterations, then it stagnated thereafter while oscillating with alternate signs. This phenomenon can be 


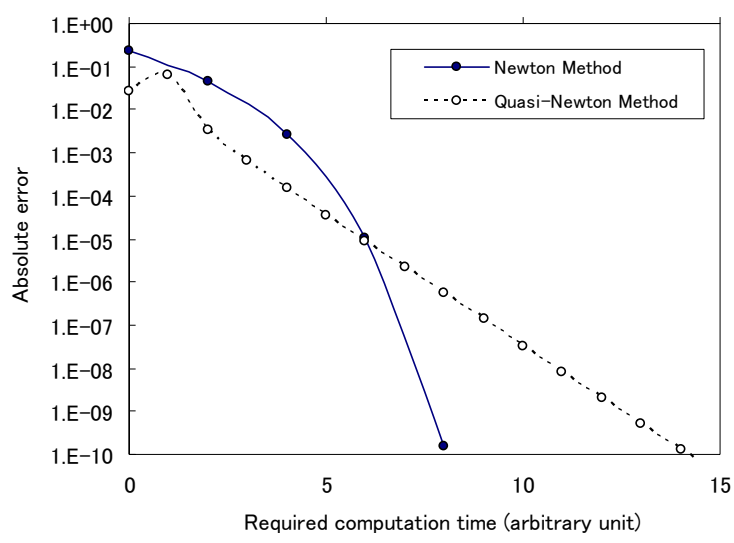

(a) $n=1$

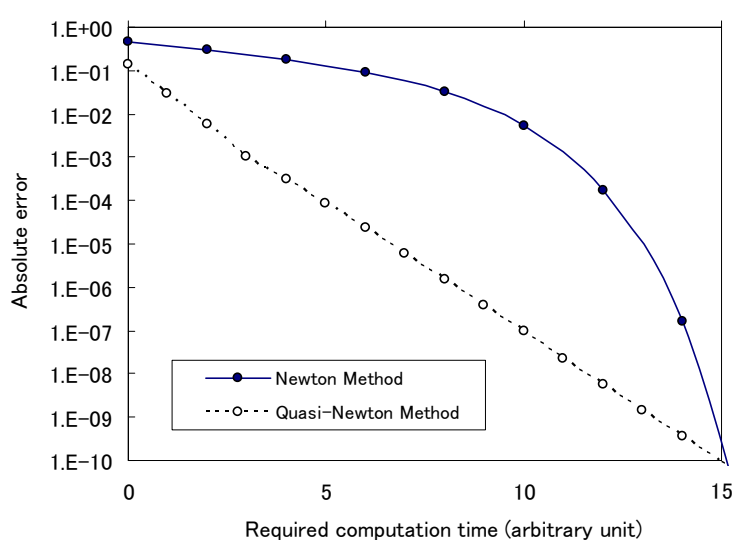

(b) $n=4$

Fig. 4 Convergence performance of the Newton and quasi-Newton methods

attributed to statistical error in the Monte Carlo calculation. In the figure, "one-sigma” error in $\lambda$ is plotted for comparison. The magnitude of the oscillation is somewhat larger than the statistical error alone, which is explainable by the numerical instability due to truncation of the denominator and numerator of Eq. (16c).

The comparison of the $k$-mode and $\alpha$-mode solutions is plotted in Fig. 6. In spite of the unrealistically large supercritical condition, which is about 20 dollars in reactivity excess, the difference in neutron spectrum is modest.

Figure 7 describes the difference in power calculated from the two mode fluxes. A large difference is seen in an energy range $1-100 \mathrm{keV}$, which overlaps that of Doppler effect. The substitution of $\alpha$-mode flux by $k$-mode leads to an overestimation of Doppler effect in any super critical condition. In the figure, statistical error, which is trivial compared to the difference expect energy range over $1 \mathrm{MeV}$, is also shown.

\section{Overall Implementation}

The neutronic calculation module of ASTERIA-FBR is composed of the main solver and several subprograms which provide the solvers with necessary updated information. The whole structure is shown in Fig. 8. The reactivity and the

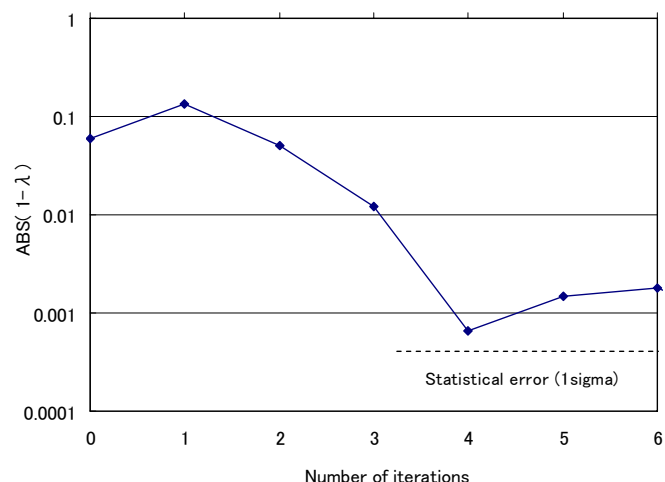

Fig. 5 Convergence performance of the quasi-Newton method in the Monte Carlo problem

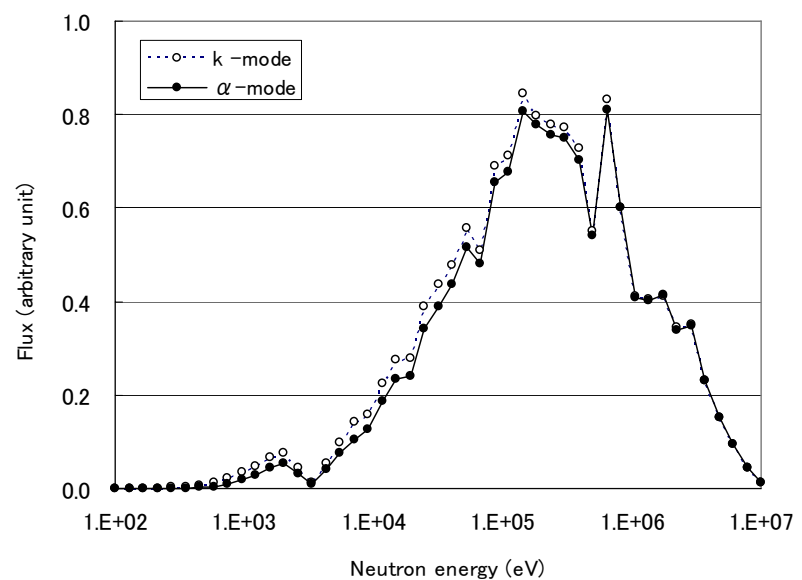

Fig. 6 Comparison of the $k$-mode and $\alpha$-mode solutions when $k=1.0635$

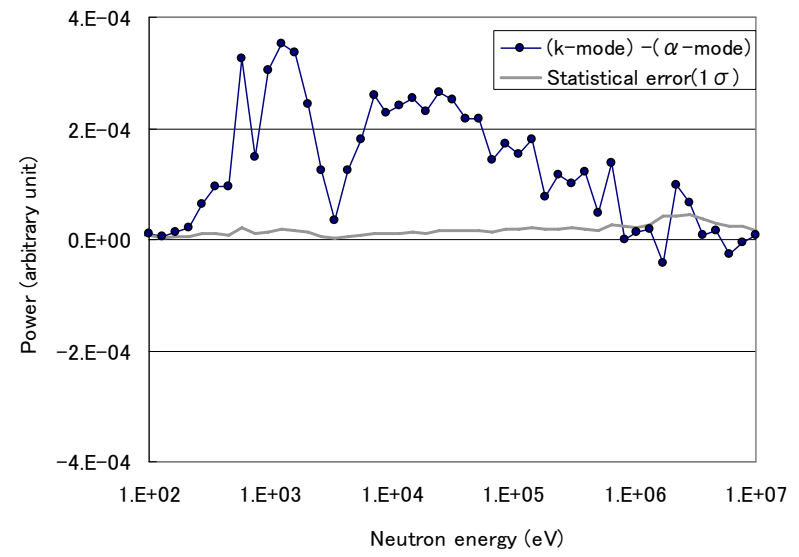

Fig. 7 Difference in power calculated from the two mode fluxes

shape function are calculated with GMVP, and the reactivity is transferred to APK code, which executes the calculation of the amplitude function at each time step.

One of the most competent features of the present code is that time-dependent dynamic parameters are treated precisely by taking into account the displacement of delayed neutron precursors contained in core debris during a core disruptive process. This feature enables us to make more realistic modeling of gaseous and volatile precursors, which occupy over $80 \%$ in delayed neutron fraction. 


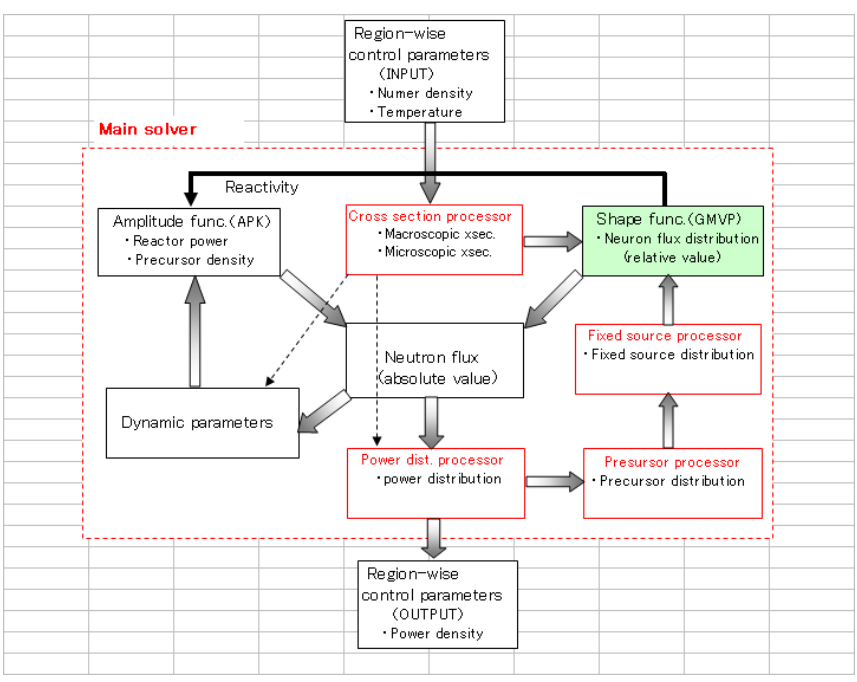

Fig. 8 The whole structure of the neutronic calculation module
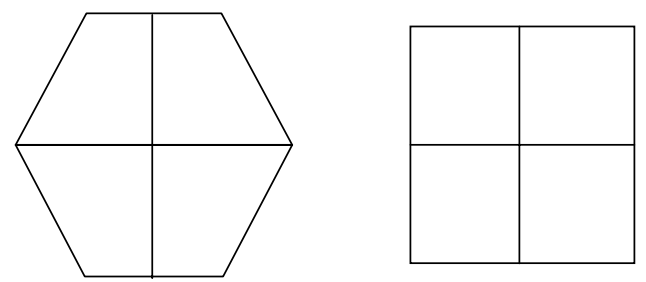

(1) Hexagonal 2x2

(2) Rectangular (equivolume) 2x2

Fig. 9 Two options for modeling a subassembly

The calculation time required in Monte Carlo is strongly dependent on shapes of tallies as well as their total numbers. Figure 9 describes two options for modeling a hexagonal subassembly. The Hexagonal 2x2 model is more favorable from the viewpoint of preservation of original geometry, while the requirement for both memory and calculation time becomes significant (Table 1).

This may be attributed to the shape of the tallies available in the code. As for GMVP, direct use of a trapezoidal tally is not allowed, so we have to use the combination of a rectangular tally and a surface tally to define the equivalent shape. This procedure requires much more computation task than a single tally does because the code has to check if a oncoming particle fulfills the condition specified by each tally at the same time. As far as GMVP is used in the system, the Rectangular 2x2 model seems to be more realistic choice at present.

\section{Conclusions}

The numerical methods to perform transient calculation based on the $\alpha$-mode with Monte Carlo code were explained. The two methods to search $\alpha$, a Newton-method-based method and an improved quasi-Newton method were newly developed and the latter was selected from the comparison of numerical test results.
Table 1 Comparison of computer resource requirements*

\begin{tabular}{ccc}
\hline & Hexagonal 2x2 & Rectangular 2x2 \\
\hline No. of axial partitions & 8 & 9 \\
$k$-mode eigenvalue & 1.04154 & 1.04731 \\
(standard deviation) & $(0.050 \%)$ & $(0.045 \%)$ \\
Memory requirement (MB) & 61.8 & 5.4 \\
Calculation time (sec) & 12757 & 151 \\
(Ratio of time) & $(84.5)$ & $(---)$ \\
\hline
\end{tabular}

* Horizontal partitions are 62x62 for the both models.

It was shown that the $k$-mode flux spectrum is softer than that of $\alpha$-mode in any supercritical condition, which leads to overestimation of Doppler effect when $k$-mode flux was used as approximation.

As for practical issues, a significant difference was observed in computer resource requirement between the regular and irregular arrangement of the tallies.

The fundamental implementation of the neutronic calculation module was completed, and application to full scale calculations is now in progress.

\section{References}

1) H. Endo, T. Ishizu, T. Yamamoto, I. Tatewaki, T. Nakajima, N. Shirakawa, T. Yokoyama, "Development of the Integrated Core Disruptive Accident Analysis Code ASTERIA-FBR, (1) Overall plan,” Proc. 2010 Annual Mtg. of the Atom. Energy Soc. of Japan, Mito, Japan, Mar. 26-28, 2010, F21 (2010), [in Japanese].

2) S. Kondo et al., "SIMMER-III : An advanced computer program for LMFBR severe accident analysis," Proc. Int. Conf. on Design and Safety Study of Advanced Nuclear Power Plants(ANP'92), Tokyo, Japan, Oct. 25-29, 1992 (1992).

3) JAEA, http://www.jaea.go.jp/jnc/zooarai/ejooarai/simmer

4) E. E. Lewis, W. E. Miller Jr., Computational methods of neutron transport, John Wiley \& Sons, New York, (1984).

5) S. A. W. Gerstl, A. Zardecki, "Discrete-ordinates finite-element method for atmospheric radiative and remote sensing," Appl. Opt., 24, 81-93 (1985).

6) S. Hamilton et al., "Negative flux fixups in discoutinuous finite element $\mathrm{S}_{\mathrm{N}}$ transport," Proc. 2009 Int. Conf. on Mathematics, Computational Methods, and Reactor Physics of Reactors(MC2009), Saratoga Springs, New York, May. 3-7, 2009, (2009).

7) G. Buckel et al., A New SIMMER-III version with improved neutronics solution algorithms, FZKA 6290, Forschungs-zentrum Karlsruhe (FZK) (1999).

8) H. Yamano et al., SIMMER-IV: A three-dimensional computer program for LMFR core disruptive accident analysis, JNC TN9400 2003-070, Japan Nuclear Cycle Development Institute (JNC) (1998).

9) K. O. Ott, R. J. Neuhold, Nuclear Reactor Dynamics, ANS, La Grange Park, USA, (1986).

10) T. Mori, M. Nakagawa, MVP/GMVP: General Purpose Monte Carlo Codes for Neutron and Photon Transport Calculations Based on Continuous Energy and Multigrain Methods, JAERI-Data/Code 94-007 (1994), [in Japanese].

11) D. E. Cullen et al., Static and dynamic criticality: are they different?, UCRL-TR-201506, USDOE (2003). 